\title{
Títeres en Pantalla: una estrategia metodológica para la expresión creativa y el aprendizaje interdisciplinar
}

On-Screen Puppets: a methodological strategy for creative expression and interdisciplinary learning

\section{Isabel Cristina Restrepo Acevedo*}

(iD) https://orcid.org/0000-0002-8235-3525

\section{Ana María Martínez Jaramillo**}

(iD) https://orcid.org/0000-0002-4281-3726

\section{Alexandra Milena Tabares García***}

(iD) https://orcid.org/0000-0002-1088-3164

\section{Investigación científica y tecnológica}

Doi: 10.17533/udea.unipluri.20.2.06

Restrepo Acevedo, I. C., Martínez Jaramillo, A. M., y Tabares García, A. M. (2020). Títeres en Pantalla: una estrategia metodológica para la expresión creativa y el aprendizaje interdisciplinar. Uni-Pluriversidad, 20(2). e2020206. doi: 10.17533/udea.unipluri.20.2.06.

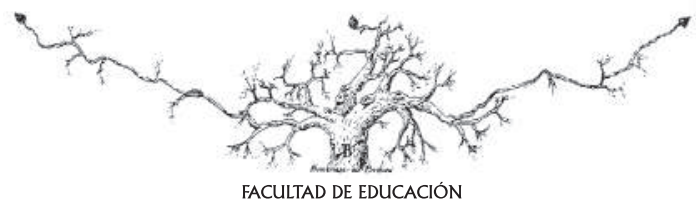

Recibido: 2020-03-25 • Aprobado: 2020-12-22

* Universidad de Antioquia. Colombia

Email: cristina.restrepo@udea.edu.co

** Universidad de Antioquia. Colombia Email: anam.martinez@udea.edu.co

*** Universidad de Antioquia. Colombia Email: alexandra.tabares@udea.edu.co 


\title{
Resumen
}

El presente artículo da cuenta de la investigación Títeres, humanos y máquinas, un circuito interdisciplinario para el aprendizaje, el intercambio cultural y la expresión creativa ${ }^{l}$. El objetivo principal fue desarrollar un Teatrino Tecnológico Híbrido (TTH), con el cual se facilitó la creación de títeres, escenarios y personajes entre niños y niñas de 4-9 años. Para ello, se trabajó bajo la metodología de investigación-creación, a través de una ruta procedimental dividida en tres etapas: Laboratorio de exploración, Laboratorio de creación y Laboratorio de implementación. De forma transversal, se desarrollaron mesas de trabajo en las que el grupo de investigadores generó la Estrategia Metodológica Títeres en Pantalla, esta partió de las premisas propuestas por el modelo pedagógico STEAM y que deriva los siguientes componentes: Un TTH, una secuencia didáctica de implementación, una página web y una versión expositiva del TTH. El proceso desarrollado en todas las etapas permitió examinar el potencial de la estrategia Títeres en Pantalla como un espacio de interacción digital que facilita un aprendizaje holístico y conectado con las realidades de los niños y niñas en un ambiente que va más allá del lineamiento de conceptos específicos disciplinares. Asimismo, la estrategia permitió que el niño actuara como sujeto con capacidades para desarollar individual y colectivamente pensamiento creativo, en la medida en que pudo poner en escena sus reflexiones en un lenguaje sensible. Lo anterior brinda espacios de enseñanza interdisciplinar en los que los niños y las niñas abordan diversos problemas desde la complejidad de los elementos que los definen, un enfoque holístico para la comprensión y resolución de problemas contemporáneos y vigentes.

Palabras clave: cocreación; metodología investigación-creación; arte; educación; tecnología; STEAM.

\begin{abstract}
This paper reports on the research Puppets, Humans and Machines, an Interdisciplinary Circuit for learning, cultural exchange, and creative expression ${ }^{2}$. The main purpose of the research was the development of a Hybrid Technological Puppet Theatre (TTH). It allowed 4 and 9 years children to create puppets, stages and characters. A Research-Creation methodology was used, through a procedural route divided into three phases: Exploration Laboratory, Creation Laboratory, and Implementation Laboratory. In a cross-sectional way, working groups were developed. In these spaces, a group of researchers developed the Puppets on Screen Methodological Strategy; it started from the premises proposed by the STEAM pedagogical model. This strategy includes the following components: TTH, didactic sequence; website and design of an expository version of the TTH. The process developed at all phases allowed to examine the potential of the Puppets on Screen strategy as a space for digital in-
\end{abstract}


teraction that provides a holistic learning connected with the realities of children, in an environment beyond the guidelines of specific disciplinary concepts. In turn, the strategy allows the child to act as a subject with capacities to produce, individually and collectively, creative thought, given that he (she) can stage his reflections in a sensitive language. The above provides spaces for interdisciplinary teaching in which, children address various problems from the complexity of the elements that define them, a holistic approach to understanding and solving contemporary and current problems.

Keywords: Cocreation; Research-Creation Methodology; Art; Education; Technology; STEAM 


\section{INTRODUCCIÓN}

Desde el año 2008, el grupo de investigación Hipertrópico, de la Facultad de Artes de la Universidad de Antioquia, ha desarrollado proyectos y creaciones que integran arte y tecnología en diversas situaciones sociales y culturales. En particular, las investigaciones han conducido a la realización de propuestas interactivas que tienen una transferencia social que va más allá de los lineamientos museográficos para ubicarse en contextos y problemáticas específicas. Dentro del tipo de población al que se ha logrado impactar se destaca, con especial interés, la infantil, en la medida en que los proyectos buscan ofrecerles un entorno propicio para la experimentación estética y el aprendizaje en diversas dimensiones cognitivas, físicas y sensibles.

Gran parte de los desarrollos del grupo han sido el resultado de un trabajo interdisciplinar en el que se articulan distintos campos del arte, así como otras áreas del conocimiento. Ejemplos de estos proyectos realizados por el grupo Hipertrópico son: la investigación Interactividad y realidad aumentada, conducente a la obra performática Entránsito, creada en asocio con el grupo GEPAR de la Universidad de Antioquia e implementada en los años 2008 y 2009 como una campaña de formación ciudadana; el videojuego Topetazo, propuesto en asociación con el grupo DIDARTE (Didáctica de las Artes), también de la UdeA, para trabajar con niños y niñas la enseñanza de las competencias ciudadanas; la tesis doctoral Tocar con los ojos, ver con las manos (Restrepo, 2014), donde se trabajaron las posibilidades y dimensiones culturales en los espacios de interacción digital con monitoreo remoto; y el proyecto de investigación Títeres poderosos, basado en una invitación realizada por el profesor Michael Nitsche del Digital World \& Image Group de la universidad Georgia Tech (Atlanta, Estados Unidos), dentro de su investigación titulada Prototyping Puppets, para facilitar la libre expresión de los niños y niñas a través de títeres desarrollados con principios básicos de STEAM (Science, Technology, Engineering, Arts and Mathematics).

Las consideraciones formales, experienciales y tecnológicas de estos desarrollos permiten señalar diferentes ejes de interés: los espacios de interacción digital con monitoreo remoto, la experiencia estética como un medio para generar procesos creativos y pedagógicos entre creadores y receptores, y la performatividad posibilitada por los ordenadores en diálogo con lo teatral. Dichos ejes fundamentan la investigación Títeres, humanos y máquinas, un circuito interdisciplinario para el aprendizaje, el intercambio cultural y la expresión creativa, acreedora del apoyo a Primeros proyectos del Comité para el Desarrollo de la Investigación en la Universidad de Antioquia, CODI, en el año 2015. Durante su ejecución se creó la Estrategia Metodológica Títeres en Pantalla para la realización de títeres e historias que permitieran la expresión creativa, el aprendizaje interdisciplinar y el intercambio cultural entre niños y niñas de básica primaria.

Conceptualmente, la investigación se articula a las reflexiones sobre las prácticas artísticas contemporáneas que superan 
el modelo convencional de producción y recepción artística, organizado estructuralmente en una relación entre artista productor de una obra y un espectador receptor pasivo, para ubicarse en un ámbito de creación artística participativa, relacional y de cocreación. De esta forma, la investigación se alimenta del concepto de arte relacional planteado por Nicolas Bourriaud (2006), en la medida en que para el proceso creativo se tiene como «horizonte teórico la esfera de las interacciones humanas y su contexto social» (p.13). Es decir, en esta investigación se propende por el desarrollo de procesos de cocreación en los que se rompe con el esquema tradicional del artista productor de obra para vincular el arte a la vida, al espectador, en una suerte de producción colectiva de conocimiento, en la que tanto productores como receptores pueden tener formas de expresión creativa y de relación interdisciplinar y social que movilizan el pensamiento sensible.

Algunos componentes que se derivan de la Estrategia Metodológica Títeres en Pantalla no solo articulan elementos virtuales y análogos, sino que permiten la puesta en escena en tiempo real, mediada por la visualización en pantalla. Por tanto, estos componentes se pueden ubicar en un tipo de propuestas interactivas denominadas Espacios de Interacción Digital con Monitoreo Remoto (Restrepo, 2014), en la medida en la que hay captación en tiempo real de las acciones de los participantes con su consecuente representación en pantalla y comparten, de esta forma, diferentes posibilidades de producción creativa con las del equipo desarrollador de la interfaz y los usuarios del sistema.

Desde la perspectiva del equipo de investigadores, al que llamaremos equipo de trabajo, la producción creativa de este tipo de espacios se entiende bajo el concepto de acto creativo, en virtud de que constituye una actividad direccionada al planteamiento de un Enunciado de interacción (Restrepo, 2014). Este enunciado está dirigido a un grupo de usuarios determinado, y es entendido como el planteamiento que detalla todos los elementos considerados para la interfaz interactiva. Las acciones de los usuarios, en cambio, son entendidas como acciones creativas, dada la libertad de su participación en el sistema al actualizar el enunciado de interacción. La acción de los usuarios particulariza acciones singulares que actualizan o le dan vida al enunciado, en una suerte de expresión espontánea, lúdica, y personal (Restrepo, 2014). En este sentido, el acto creativo es más un proceso de pensamiento, orientado a generar un enunciado de interacción con una intencionalidad social y cultural determinada, mientras que las acciones creativas permiten la emergencia de expresiones más singulares, sean individuales o colectivas, de carácter empírico y desinteresado de lineamientos socioculturales por parte de los usuarios.

De otro lado, es importante señalar cómo la participación de los usuarios en este tipo de espacios interactivos los lleva a tener una acción performática, cercana a la puesta en escena teatral, en tanto, quienes interactúan, lo hacen integrando su cuerpo y su mente en una expresión que bien puede denominarse «dramática», en el sentido de que combina gestualidad, movimiento y trabajo sonoro y vocal. Lo anterior puede alimentarse de consideraciones relacionadas con una suerte de performatividad posibilitada por los ordenadores en relación con el teatro. Esta es una propuesta de la teórica Brenda Laurel (1993) en su libro Computer as theater, quien invita a pensar en las ac- 
tividades humanas mediadas por computador (human-computer activity) en términos teatrales, dado que en las puestas en escena se dan unas relaciones entre las representaciones y la realidad que están establecidas desde perspectivas teóricas y prácticas.

Por tanto, en la investigación se valora, con especial énfasis, la potencia del teatro como lenguaje «que consiste en la representación o actuación de historias en frente del público, usando para estos fines el habla, los gestos, la mímica, la danza, la música y otros elementos» (Reina, 2009, p.1). De forma particular, dentro de las consideraciones de uno de nuestros objetivos, que es la creación de un Teatrino Tecnológico Híbrido (TTH), es de gran interés la representación con títeres ${ }^{3}$, dado que en este tipo de representaciones se integran distintos materiales y técnicas teatrales para representar historias (Guerra, 2008). Entre sus potencialidades, Cárdenas (2014) señala que el teatro de títeres le ofrece al actor la oportunidad de tener control sobre los medios creativos tanto artísticos como de producción y le permite una ilimitada utilización de materiales para su construcción. A su vez, le ofrece la libertad de hablar y actuar sin mayores restricciones. Por último, el autor señala que esta expresión artística se presenta como una forma de vincular las experiencias cotidianas con el reino de la fantasía pura.

Al analizar la capacidad de los títeres como lenguaje expresivo se observa su potencialidad para el trabajo creativo y pedagógico con niños y niñas. Según señala Vigotsky (2009), dado que las representaciones de títeres parten de acciones realizadas por los niños y niñas, se facilita la integración de las creaciones desarrolladas desde el arte con sus experiencias cotidianas. En este sentido, los títeres se presentan para esta población como un recurso artístico que le permite al niño expresar, inventar y narrar historias de forma lúdica, que conllevan a aprendizajes interdisciplinares y variados como la creatividad y el aumento de la imaginación (Herrero, 2017).

Es importante señalar cómo el TTH y, en general, el enunciado de interacción, como resultados de la investigación, se alimentan de las tecnologías digitales que ofrecen hoy en día diferentes maneras y herramientas para la creación de lugares de interacción que integran componentes físicos y digitales en un mismo espacio de visualización - la pantalla- $y$, además, implican la relación de los individuos con los contenidos virtuales: audiovisual, sonoro, textual, táctil, etc. Estos nexos entre las máquinas y los humanos permiten la emergencia de nuevas posibilidades para que el cuerpo y sus distintos órganos posean otras maneras de expresión, creación y aprendizaje (Restrepo, 2014) y faciliten, a su vez, el desarrollo de las diferentes dimensiones del ser humano: cognitiva, socioafectiva, comunicativa, corporal y creativa.

Esto hace visible las potencialidades de generar procesos pedagógicos con títeres y tecnologías digitales en la enseñanza-aprendizaje; y procesos que sean más holísticos y contextualizados a las realidades, problemáticas y necesidades de los niños y sus entornos. Por tanto, partimos de las premisas propuestas en el modelo educativo STEAM (Science, Technology, Engineering, Art and Mathematics) donde se busca la integralidad del aprendizaje a través del arte y la creatividad. Este acrónimo se extendió en Corea durante el año 2011, tras considerar la importancia de involucrar a las áreas sociales en la interdisciplinariedad inicialmente 
planteada en los años 90 por Estados Unidos a través del STEM (Science, Technology, Engineering and Mathematics) (Carmona-Mesa, Cardona, Castrillón-Yepes, 2020). Esta integración entre STEM y Art se debe a que a la educación le corresponde preparar a los niños y jóvenes y "para resolver los problemas apremiantes del mundo a través de la innovación, la creatividad, el pensamiento crítico, la comunicación efectiva, la colaboración y, en última instancia, el conocimiento nuevo" (Quiley y Herro, 2016, p. 410, en Ortiz-Revilla, Jairo \& Greca, Ileana $\&$ Arriassecq e Irene, 2018, p.824), además de la importancia de la expresión creativa en el desarrollo del ser humano.
Bajo estas perspectivas, el grupo de investigación Hipertrópico se interesó por diseñar una propuesta pedagógica, artística, tecnológica, interactiva e interdisciplinar para la generación de experiencias híbridas, entendidas como aquellas que integran componentes físicos y digitales. En particular, se busca que estas experiencias permitan a los niños y niñas, entre los cuatro y nueve años, expresarse de forma creativa y, a su vez, desarrollar aprendizajes significativos de las diferentes disciplinas STEAM. De esta forma, se pretende que los niños y niñas desarrollen acciones intencionadas bajo un Enunciado de Interacción propuesto por el equipo de trabajo como una Estrategia Metodológica denominada Títeres en Pantalla.

\section{Metodología}

口

El arte, como disciplina sobre la cual se fundamenta el desarrollo de la investigación que da lugar al presente artículo, despliega, al igual que el diseño y la arquitectura, la investigación-creación como el paradigma que concibe la generación de nuevo conocimiento a través del proceso de creación. Como lo exponen las profesoras Ballesteros y Beltrán (2018), tras la aplicación de técnicas investigativas pertinentes, se manifiesta y estructura «el conocimiento tácito que se inscribe en los artefactos y procesos de la creación para generar transformaciones en el entorno actual del ser humano» y se responde a in- quietudes oportunas «para el ámbito de la investigación en el mundo de la creación en un contexto político y académico» (p. 28). Así, al ejecutar la investigación Títeres, humanos $y$ máquinas, se atienden las particularidades del proceso investigativo y se proyecta su relevancia en el campo de estudio al articular métodos e instrumentos para la investigación que, provenientes de otras disciplinas, contribuyen a la documentación, análisis y circulación del conocimiento emergente, a partir de los cruces, retroalimentaciones, transformaciones e impactos de las actividades o acciones presupuestadas.

\section{Participantes}

El desarrollo de la presente investigación contó con la participación de un grupo de investigadores de diferentes disciplinas denominado Equipo de trabajo, conformado por: tres docentes de la Facultad de Artes, un estudiante en formación del posgrado en
Maestría en Ingeniería, cinco estudiantes en formación de pregrado del programa Artes Visuales y una estudiante en Maestría en Estudios en Infancias, quien a su vez es docente de la Facultad de Educación. Este equipo estuvo a cargo del desarrollo del plantea- 
miento de un Enunciado de interacción de la Estrategia Metodológica Títeres en Pantalla, es decir, fue el encargado de desarrollar los objetivos de la investigación y, a la vez, de diseñar las estrategias para tal fin.

Otro perfil de participantes que contribuyeron con aportes al diseño del Enunciado de interacción fueron los Colaboradores académicos, quienes brindaron retroalimentación desde su experticia técnica, académica, investigativa y pedagógica en los distintos momentos de la investigación. De forma adicional, se contó con la participación de Colaboradores creativos - niños de edades que oscilaban entre los cuatro y nueve años-, quienes realizaron personajes y escenarios que fueron digitalizados para ser integrados a la creación de software.

Teniendo en cuenta las consideraciones éticas con la población infantil, esta investigación diseñó formatos de consentimiento para los padres o adultos acudientes, así como el asentimiento para los Colaboradores creativos - niños y niñas-, de acuerdo con las consideraciones ético-metodológicas desarrolladas por la investigadora Maribel Barreto en las que propone la comprensión de los niños y niñas como sujetos con derecho a ser consultados sobre su deseo e interés en participar del proceso (Barreto, 2011). En particular, la autora señala que para la investigación con niños y niñas:

Aunque un niño o niña puede no ser legalmente competente, debe dar el asentimiento antes de ser involucrado en un estudio; esto significa que debe saber que puede aceptar participar de la investigación y también que puede retirarse en cualquier momento si lo desea, sin que ello implique un detrimento en su cuidado; de igual manera, ha de comprender cuál es su papel, saber qué debe hacer si elige participar, saber qué suce- derá con los datos que se generen, y conocer cuáles son los riesgos que tiene si decide participar. (p.645)

En el consentimiento se da a conocer el nombre de la investigación, persona a cargo, párrafo descriptivo de los objetivos, el propósito de los talleres, la autorización para el registro de imágenes con usos sólo académicos y, finalmente, el permiso para ser firmado con número de cédula por el adulto, atendiendo al ejercicio de la Patria Potestad establecido en el Código Civil colombiano en su artículo 228, el artículo 24 del Decreto 2820 de 1974 y la Ley de Infancia y Adolescencia. Por otro lado, en el asentimiento se le cuenta al niño, de manera gráfica, en qué consiste la investigación y los talleres y, al final, se le consulta si desea hacer parte de esta con un gesto gráfico o escrito, dependiendo de la edad.

En general, para todos los participantes de la investigación y de la estrategia se abogó por el uso de «la imaginación como elemento conductor de la creatividad» (Daza, 2009, p.89). Sin embargo, este uso de la imaginación fue conjugado, para el caso del equipo de trabajo, con la ayuda de los colaboradores, de una manera objetiva y racional que permitiera encaminar la incertidumbre que genera el acto creativo para alcanzar los objetivos y resultados de la investigación. Esto delimitó las coordenadas para la Estrategia Metodológica Títeres en Pantalla. Mientras tanto, el uso de la imaginación en los participantes que actualizaron el enunciado fue más espontáneo y respondió a fines más lúdicos y de aprendizaje vivencial; esto se basa en la experiencia de su participación en talleres de creación o en la interacción en otros procesos de la Estrategia. 


\section{MÉTodo}

口

Títeres, humanos y máquinas se ejecutó tras la conformación de Mesas de trabajo que se entienden como encuentros periódicos entre los investigadores, estudiantes en formación e integrantes de los semilleros vinculados a la investigación. En estas se realizaron ejercicios transversales de conceptualización, experimentación, diseño y proyección técnica, pedagógica y creativa, que tuvieron como orientación la siguiente ruta procedimental dividida en tres laboratorios: Laboratorio de exploración, Laboratorio de creación y Laboratorio de implementación.

Los laboratorios son entendidos como las experiencias diseñadas para un grupo específico de participantes, con los cuales se procura el intercambio de saberes, la creación, apropiación, discusión y evaluación de conceptos, ideas y prototipos articulados a la investigación-creación. Dicho de otra manera, los laboratorios son escenarios relacionales dinámicos, planteados como ambientes creativos para el despliegue de acciones o discusiones que propician la reelaboración de imaginarios y el fortalecimiento de premisas investigativas.

En el Laboratorio de exploración, el equipo de trabajo indaga las variables convergentes o divergentes de cada acción trazada para la investigación, con el propósito de esbozar propuestas estratégicas para la realización eficaz de actividades orientadas al alcance de los objetivos. En el Laboratorio de creación, el equipo de trabajo dispone, para los demás participantes de la investigación, los planteamientos perfilados en el Laboratorio de exploración y busca estimular en los asistentes la concepción de ideas y sus posibles materializaciones. En este laboratorio, cada miembro del equipo de trabajo debe atender a una tarea específica, ya sea en la dirección de un momento de la experiencia o en la recolección de información a partir de ejercicios de observación. En el Laboratorio de implementación se ponen a prueba los prototipados y los diseños de la propuesta metodológica, definida en las Mesas de trabajo, para analizar su pertinencia pedagógica, su efectividad técnica y la apropiación que de ella tienen los niños participantes y el público asistente. De esta manera, en las Mesas de trabajo el equipo revisa, discute, cruza, contrasta y relaciona acciones realizadas en cada experiencia de laboratorio, es decir, como un procedimiento hermenéutico de revisión constante, de ir $\mathrm{y}$ volver en cada uno de los procesos.

\section{Laboratorio de exploración.}

El Laboratorio de exploración consistió en la experimentación con juegos de mesa diseñados para la construcción de historias, personajes y escenarios. Uno de ellos fue La caja de juegos iA crear historias! de Ani Laval y el libro interactivo Animalario universal del profesor Revillod: Almanaque ilustrado de la fauna mundial de Miguel Murugarren (2003). Esta experimentación propició la formulación de inquietudes investigativas como: ¿el TTH deberá disponer una historia inicial para invitar a la interacción y a la construcción de otras historias posibles?, ¿el TTH deberá tener un repositorio de historias al cual puedan recurrir los nuevos interactores?, si la disposición de elementos gráficos no limita la creatividad y facilita el proceso de interacción, entonces, ¿el TTH deberá contener personajes y escenarios prediseñados? 
La heterogeneidad de respuestas emergentes a lo largo del proceso de investigación-creación, motivadas por estos cuestionamientos iniciales, determinó la toma de decisiones significativas para el diseño del TTH y de la estrategia metodológica.

\section{Laboratorio de creación}

Luego del análisis de las preguntas anteriores, el equipo de trabajo consideró la necesidad de diseñar un Laboratorio de creación, con la idea de estructurar talleres $^{4}$ presenciales dirigidos a niños, para la creación de historias, escenarios y personajes como insumos para el desarrollo del software. Así, fue necesario que el equipo de trabajo se dividiera en tres semilleros enfocados en los componentes: programación, pedagógico y audiovisual. Debido a la complejidad que requería cada uno, se integraron al proceso estudiantes provenientes de distintas áreas de formación, con el objetivo de profundizar en elementos conceptuales y creativos requeridos para el cumplimiento de los objetivos, en particular para la creación del TTH. La dinámica de trabajo de la investigación se dio entre Mesas de trabajo por semilleros, con otras colectivas donde se reunía todo el equipo de trabajo con miras a generar una estructura integral para el TTH. A continuación, se detallan los objetivos y actividades de cada semillero:

Semillero Pedagógico: se centró en la conceptualización, diseño y ejecución de los talleres para la cocreación de algunos elementos requeridos para el desarrollo de la estrategia metodológica en general y elaboró los instrumentos para la recolección, documentación y análisis de la información, así como de los instrumentos para dar cumplimiento a las consideraciones éticas.

Semillero Programación: su principal objetivo fue el desarrollo técnico de la plataforma interactiva para el proyecto, mediante la exploración y desarrollo de un código propio, así como hardware que facilitara la interacción para la puesta en escena de los niños.

Semillero Audiovisual: se ocupó de explorar y aplicar metodologías de registro que permitieran la creación, la documentación, la investigación y la socialización de las distintas etapas del proceso a través de la captura de fotografías y videos.

\section{Figura 1}

Creación de historias - Talleres MUUA. Imagen cortesía del grupo de investigación Hipertrópico, UdeA.

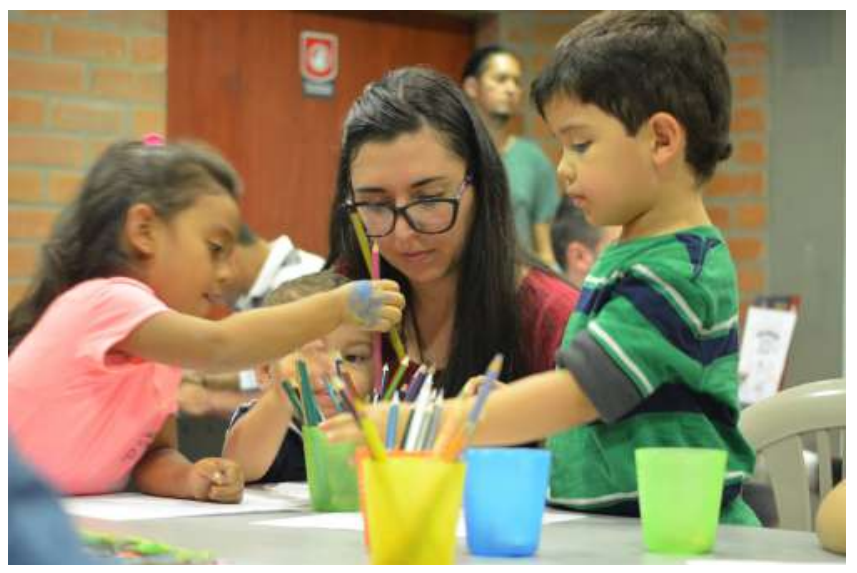


Para llevar a cabo los talleres del Laboratorio de creación, se realizó una alianza con el Museo Universitario Universidad de Antioquia (MUUA), en el marco de su espacio Títeres en recreo. Fue así como se creó la secuencia didáctica para once sesiones, fundamentada en la expresión y la capacidad creadora, donde la producción de los niños no es una obra de arte, sino un juego en el cual se satisface una necesidad de expresión, una liberación (Stern, 1977, 2008), donde el niño se identifica con sus propias experiencias y logra expresar sus vivencias desde su sensibilidad estética (Lowenfeld, 1961).

De igual manera, se diseñaron acciones intencionadas para posibilitar el acer- camiento de los niños a nociones de conceptos de las diferentes disciplinas STEAM mediante los procesos de experimentación y creación. De acuerdo con Cilleruelo y Zubiaga (2014), la incorporación de las artes en una corriente educativa, enfocada inicialmente en la interdisciplinariedad acontecida entre la ciencia, la tecnología, la ingeniería y las matemáticas (STEM), hace que los aprendizajes deriven del abordaje de «problemas deseados, de las ganas de saber», de tal manera que «la curiosidad se convierte en motor y guía del conocimiento, un punto de partida para la exploración de diferentes soluciones en una búsqueda permanente de la satisfacción personal» (p. 2).

\section{Figura 2}

Recorrido por la sala de Historia Natural del MUUA. Imagen cortesía del grupo de investigación Hipertrópico, UdeA

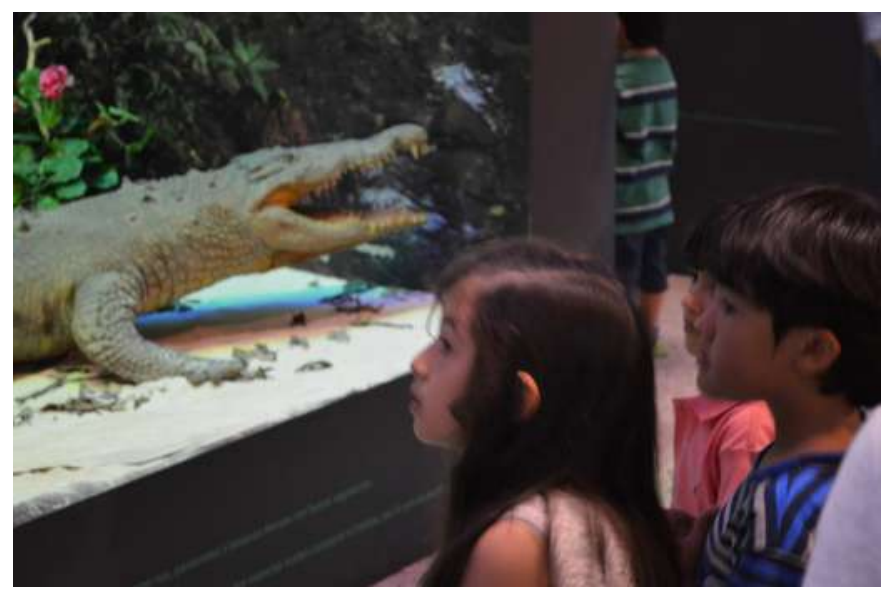

Para identificar la manera en que podrían suceder los aprendizajes vinculados a cada una de las disciplinas convergentes en STEAM, se diseñó un formato de observación, el cual contenía unas unidades de análisis basadas en los indicadores de las diferentes áreas propuestas por el Ministerio de Educación Nacional de Colombia (MEN), de acuerdo con las edades de los niños. Este formato fue diligenciado por cada uno de los miembros del semillero pedagógico y allí realizaron una breve descripción.

La planeación de los talleres apuntó a la generación y recolección de insumos para la construcción del TTH. Esta se acopló a las necesidades y capacidades que surgieron en su desarrollo y se llevó a cabo mediante un formato llamado Diseño de encuentros, en el cual se hizo la descripción de la fecha 
de realización, el objetivo general, los objetivos específicos, las actividades (saludo, actividad de provocación, actividad central y actividad de cierre), descripción de las actividades, materiales y responsables.

Aunque se proyectó para once sesiones, el Laboratorio de creación se realizó en siete sesiones que iniciaron con un recorrido por la colección de Historia Natural del MUUA, con el fin de estimular su capacidad de observación y despertar el interés mediante preguntas intencionadas desde la metodología STEAM. Para la creación de historias, escenarios y personajes se integró la utilización de materiales fungibles, naturales y reutilizables, así como la expe- rimentación sonora. En las últimas sesiones se exploró la mecánica de interacción con el TTH, compuesto por los personajes y escenarios creados con anterioridad, con miras a permitir que la acción performática de la última sesión emergiera con espontaneidad desde la experiencia construida en las sesiones anteriores y con las ideas que surgieron en la experiencia en vivo. Los niños y niñas realizaron, a través del TTH, una presentación performática final con los personajes y escenarios que habían creado en los encuentros pasados. Los espectadores de la presentación fueron los adultos acudientes de los niños y niñas, y el equipo de trabajo y colaboradores del MUUA fungieron como mediadores.

Figura 3

Exploración sonora- talleres MUUA. Imagen cortesía del grupo de investigación Hipertrópico, UdeA.

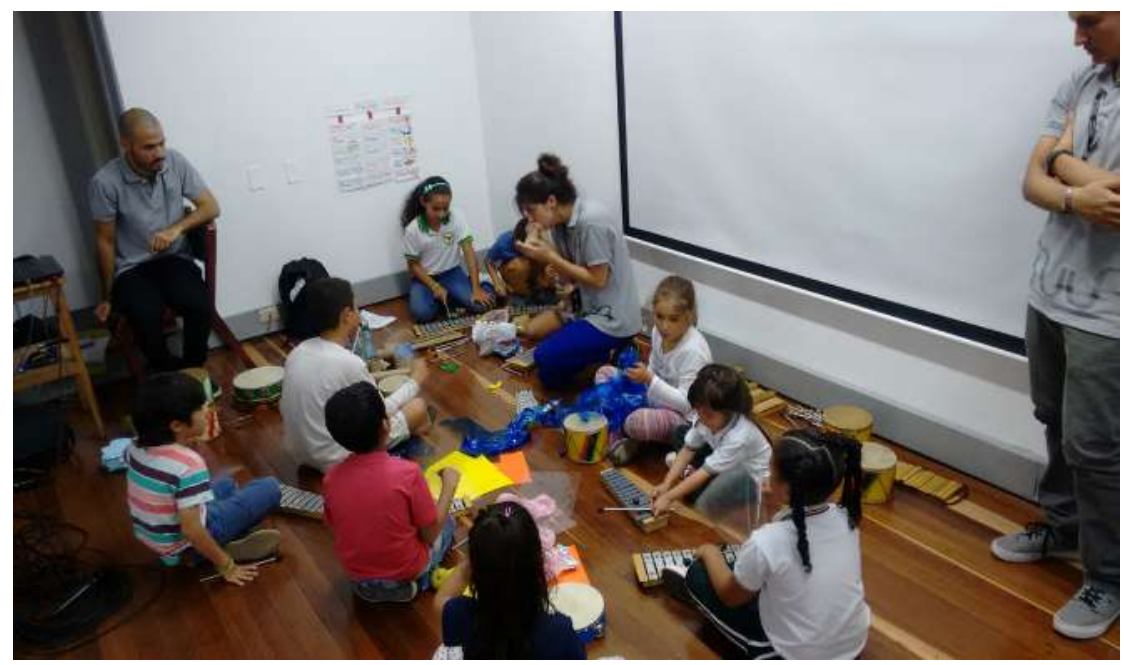

\section{TTH versión Beta}

En la etapa de Laboratorio de exploración, el equipo de trabajo generó una lluvia de ideas sobre lo que podrían ser los componentes del teatrino. Así, se propuso que el escenario virtual estuviera compuesto por un banco de objetos digitalizados, como partes del cuerpo y escenarios predeterminados, con el fin de que los niños y niñas armaran sus personajes y crearan sus historias. En la etapa inicial del Laboratorio de creación se visualizó la posibilidad de construir los títeres-controladores con palitos de paleta. Sin embargo, la idea fue evolucionando hacia el diseño de piezas ensamblables de madera aglomerada cortadas con láser. 


\section{Figura 4}

Ideas iniciales sobre la plataforma. Imagen cortesía del Grupo de Investigación Hipertrópico, UdeA.

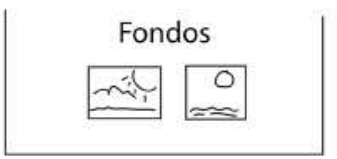

Eje temático

Performance inicial $=$ Invitación

Canal YouTube

Sitio web

Video

Resumen

Registros

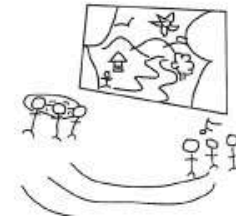

Posteriormente, se exploraron herramientas para el procesamiento digital como OpenCV (software y librería de visión artificial que se puede integrar a distintos lenguajes de programación; se utilizó, para este caso, Processing) 5 . Durante estos procesos se vislumbraron diferentes posibilidades que podrían utilizarse para el desarrollo de la plataforma, como el reconocimiento de marcadores para generar movimiento con las diferentes poses de un títere, aplicando cambios consecuentes en la representación del avatar en pantalla y cambios en los escenarios e integración de elementos dinámicos, por ejemplo, el clima.

Partiendo de lo anterior, el Semillero de Programación comenzó a trabajar en el desarrollo del TTH y desarrolló aspectos tales como: estructura modular para crear personajes (esqueleto), control del número de miembros y tamaño del personaje, texturas para los personajes, caracterización de los personajes (con los distintos diseños definidos en el taller), elementos físicos que afectan al personaje (como la gravedad y la colisión) y movimientos de los personajes
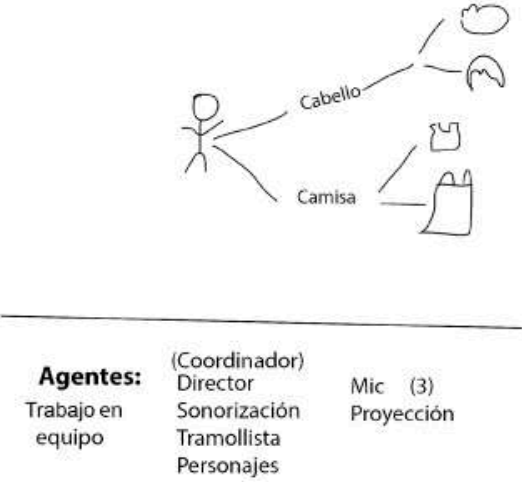

(como su cabeza, boca sincronizada con voz de actor, ojos vivos...). Se tuvo en cuenta los bocetos planteados al comienzo.

Sin embargo, durante la realización de los talleres, se identificaron aspectos que obligaron a realizar ajustes a estas ideas y a las exploraciones iniciales. Por un lado, se reconoció la potencialidad de incluir las producciones de los niños, en vez de tener personajes y escenarios predeterminados. Por otro lado, durante el desarrollo del software, surgieron dificultades como la falta de tiempo para el diseño de mecanismos de captura, a partir de varios puntos que contenía el títere, por lo que fue necesario descartar la idea del movimiento articulado y fortalecer la captura mediante la cámara web por medio de marcadores de colores con las siluetas de los personajes y el uso del títere varilla ${ }^{6}$. Lo anterior se logró mediante el procesamiento digital de imagen, utilizando la opción de umbral del color. Así, la plataforma permitió el reconocimiento de cuatro colores a través de la cámara web, y el control y posicionamiento en pantalla de los personajes digitalizados o avatares. Los avatares ${ }^{7}$ fueron las imágenes 
vectorizadas de los personajes elaborados por los niños y niñas. En esta misma línea, se integraron dos escenarios, el bosque y el desierto, también realizados por los niños y niñas, que fueron controlados a través del teclado del computador.

Por otro lado, en los últimos talleres del Laboratorio de creación, se presentaron inconvenientes con diferentes elementos como la cámara, los controladores de color, la mesa, la pantalla o proyector, entre otros. Esto condujo al diseño de una estructura que permitiera acoplar los distintos elementos de hardware y software para facilitar un mejor funcionamiento del TTH.

\section{Figura 5}

Imagen de la pantalla en la que se muestra una parte del código y la imagen resultante donde se combinan el escenario y los personajes. Imagen cortesía del Grupo de investigación Hipertrópico, UdeA.

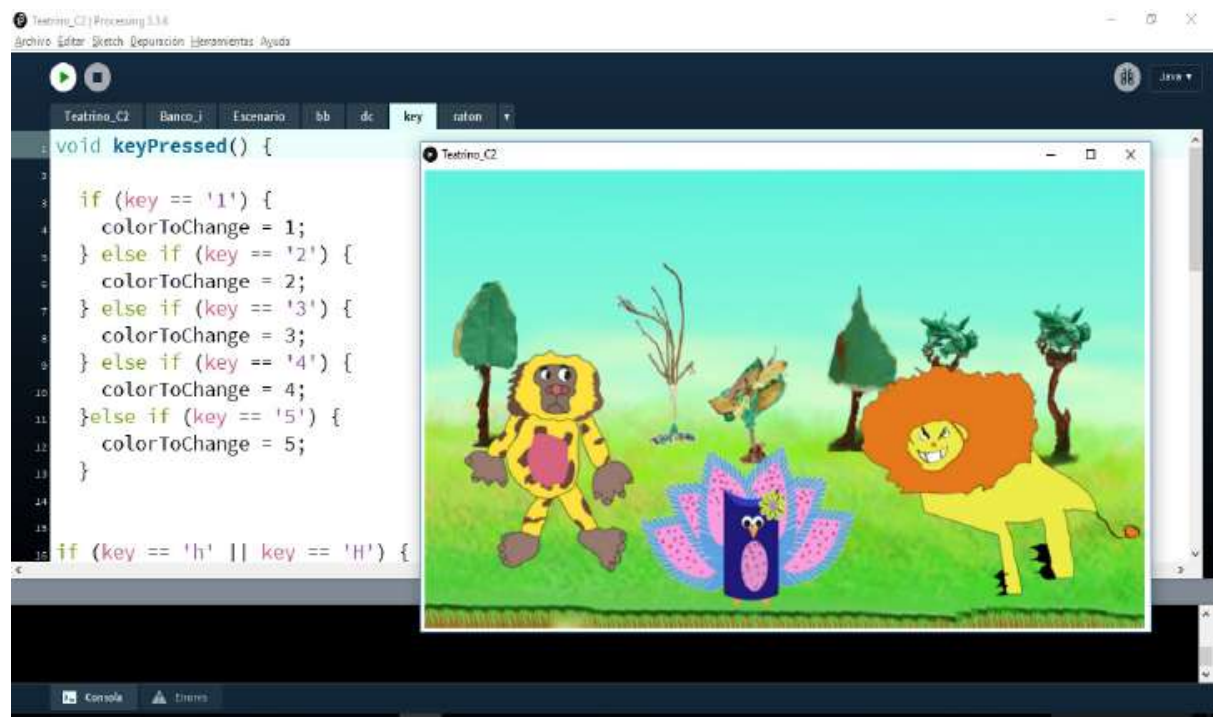

Luego del Laboratorio de creación, se realizó un proceso de evaluación con el equipo de trabajo sobre diferentes aspectos del diseño y la implementación del TTH. Los resultados permitieron trazar una ruta para la realización de ajustes e implementación del teatrino y, a su vez, expandir las formas de aprendizaje, intercambio y expresión creativa al formato de página web y de versión expositiva.

\section{Laboratorio de Implementación}

Este laboratorio partió de la necesidad de implementar las creaciones en un escenario escolar, así como con otras edades den- tro de la básica primaria. Para lo anterior, se pensó en posibles instituciones públicas de la ciudad de Medellín o en proyectos aliados con los cuales se pudiese tener un profesor de enlace que facilitaran el trabajo con los niños y niñas. Esto fue posible gracias al vínculo con la investigación Laboratorio de Territorio, Ciudadanía y $\mathrm{Paz}^{8}$, mediante la cual se realizó la implementación en la Institución Educativa Villa Flora - en el barrio Robledo de Medellín - con un grupo piloto de dieciséis niños y niñas de los grados $4 .^{\circ} \mathrm{y}$ 5. ${ }^{\circ}$ de primaria.

Al tener claras las variables y las premisas base del objetivo de investigación, el 
equipo de trabajo desarrolló un enunciado de interacción compuesto de cuatro componentes, a saber: secuencia didáctica de talleres, TTH, página web, y versión expositiva del TTH.

De forma particular, la didáctica para los talleres en Villa Flora se desarrolló en seis sesiones, enfocadas hacia la construcción y realización de narrativas, cartografías y personajes mediante actividades asociadas al STEAM, a partir de preguntas movilizadoras sobre el contexto en que viven los niños y niñas. Los resultados de cada una de las sesiones fueron llevados al software del TTH y fueron integrados a la página web del proyecto Títeres, humanos y máquinas y a la versión instalativa. Para tal fin, se diseñó una secuencia didáctica para la implementación del TTH.

\section{Figura 6}

Mapa del territorio realizado por los niños que luego se transformaría en el escenario de su historia. Talleres I.E. Villa Flora. Imagen cortesía de la investigación Laboratorio de Territorio, Ciudadanía y Paz, desarrollada por el INER y el grupo de investigación Hipertrópico, de la UdeA.

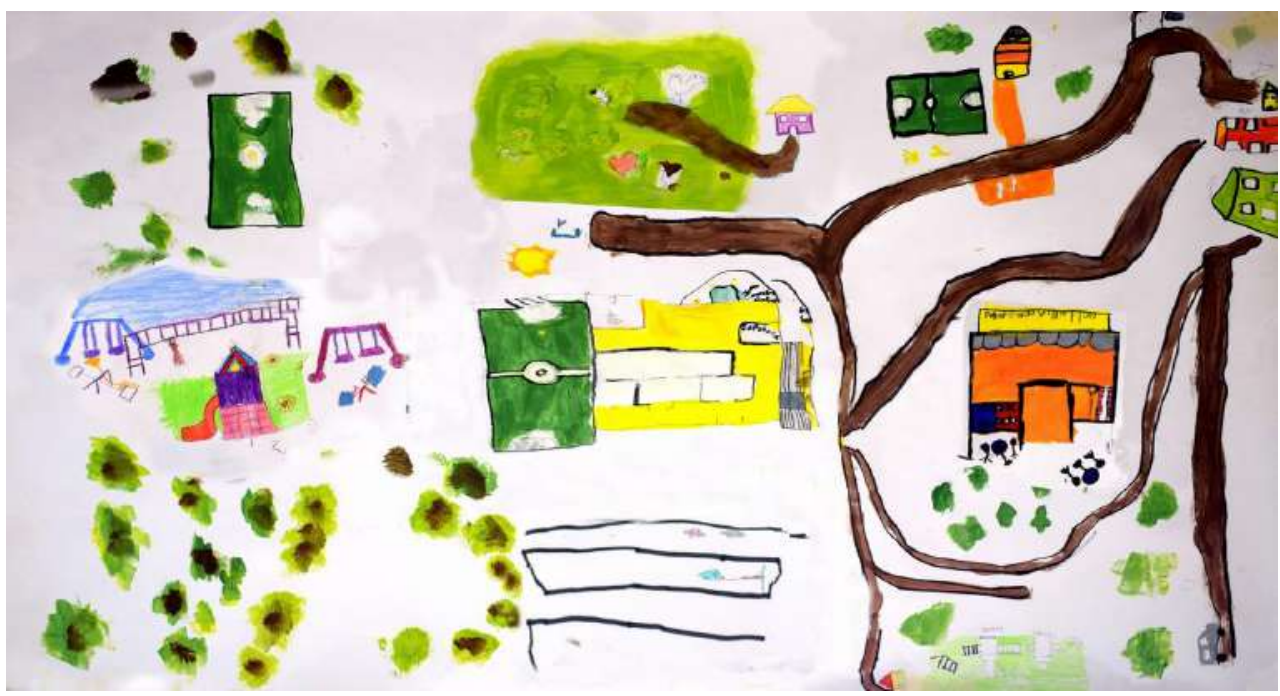

\section{Desarrollo de los encuentros.}

Los encuentros en la I. E. Villa Flora implicaron tres etapas fundamentales: introducción, creación e implementación de resultados. Con la introducción se establecieron las premisas de trabajo y acuerdos, consentimientos y asentimientos; también se hizo un diagnóstico de saberes. En la creación se dieron los elementos para favorecer la construcción de historias, personajes y escenarios asociados al territorio que habitan. Luego, se estableció un tiempo para la preparación y presentación de resultados finales, lo que implicó que los niños y niñas realizaran una puesta en escena de sus creaciones para sus compañeros de los talleres. Al final, se generó un espacio de reflexión sobre todo el proceso. De forma transversal, todas las sesiones brindaron espacios para desarrollar contenidos y aprestamientos relacionados con posibilidades de mediación para la paz y la ciudadanía y de implementación tecnológica y expresión creativa para hacer, de este modo, un énfasis en cumplir con la visión integral de la metodología STEAM. 


\section{Figura 7}

Realización de la puesta en escena. Talleres I.E. Villa Flora. Imagen cortesía de la investigación Laboratorio de Territorio, Ciudadanía y Paz, desarrollada por el INER y el grupo de investigación Hipertrópico de la Facultad de Artes, ambos de la UdeA.

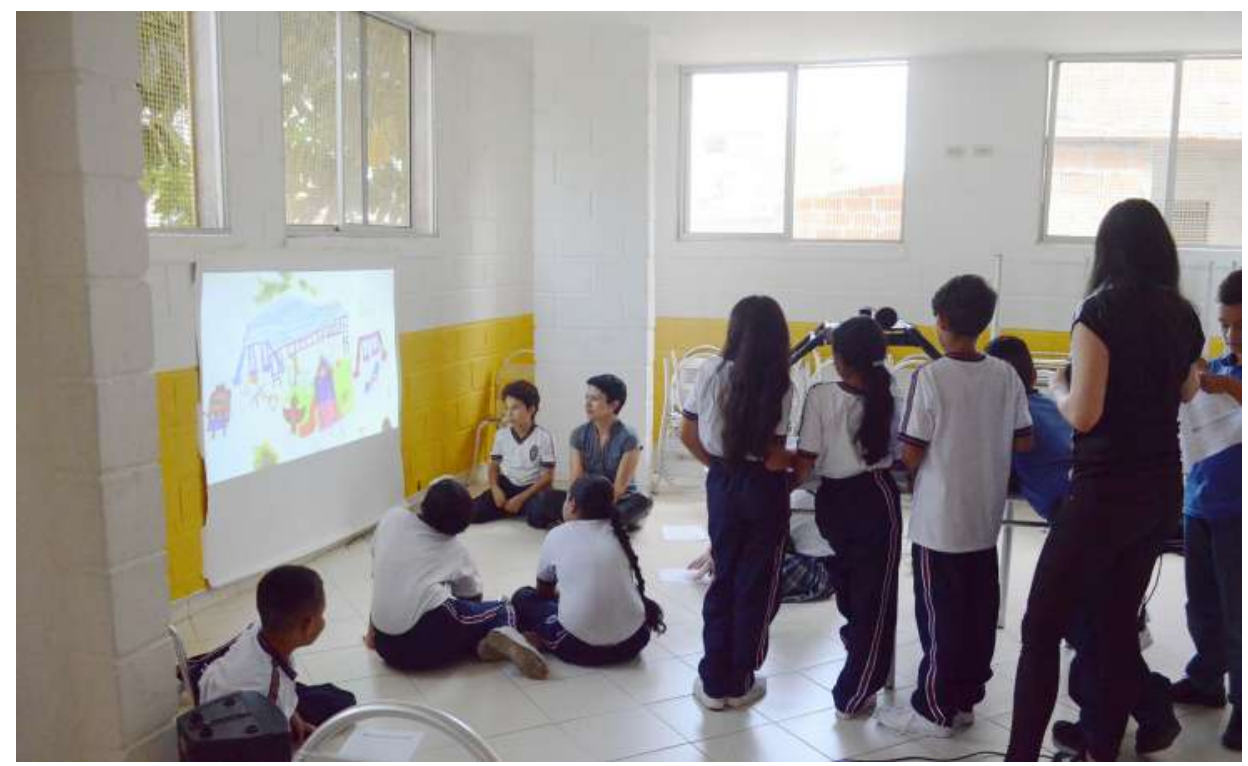

\section{RESULTADOS DEL ENUNCIADO DE INTERACCIÓN A LA ESTRATEGIA METOdOLÓgICA TÍTERES EN PANTALLA}

Las reflexiones sobre el enunciado de interacción con el cual se podría llevar a cabo el desarrollo de una plataforma para el intercambio cultural y la expresión creativa bajo la metodología STEAM, generó la necesidad de pensar en varios componentes que facilitasen dinámicas de participación sincrónicas y asincrónicas, es decir, en tiempo real o diferidas. En este sentido, el planteamiento del enunciado de interacción se diseñó en cada uno de sus componentes de tal manera que se facilitara la interacción entre usuarios, así como entre usuarios y el sistema, es decir, entre humanos y máquinas.

Con base en las consideraciones anteriores, se creó como enunciado de interacción la Estrategia Metodológica Títeres en
Pantalla. Esta está compuesta por el TTH, la secuencia didáctica de talleres de implementación del TTH, la página web, y la propuesta expositiva del TTH. Si bien gran parte de los componentes están pensados para niños, niñas y docentes, existe la posibilidad de tener otros públicos. A continuación, se detallan los componentes de la Estrategia:

\section{Teatrino Tecnológico Híbrido (TTH):} Es el dispositivo que permite la interacción en tiempo real a partir de la secuencia didáctica implementada para los talleres o a través de las instrucciones dadas en la versión expositiva que se haga del proyecto. Los componentes finales del TTH son: software titulado bajo este mismo nombre e inscrito ante la oficina de derechos de autor, computador, cámara web, video proyector, es- 
tructura tubular de teatrino y controladores. En términos de software se logró desarrollar una versión estable, fácil de instalar, y con la posibilidad de personalizar de uno (1) a seis (6) escenarios para las historias, a través de una estrategia de división reticular del espacio asignado para la imagen de fondo. Para facilitar la implementación se desarrolló un manual de instalación y uso.

Los equipos mínimos en términos de hardware y software fueron pensados de forma integral, considerando el desarrollo de componentes de interactividad digital con monitoreo remoto en tiempo real a partir de la captación de colores, así como la consideración de que el software pueda ser descargado por cualquier persona interesada en implementar el proyecto.

Adicionalmente, se elaboró una estructura tubular negra de fácil ensamble que sirve para soportar la cámara web en la parte superior y el fondo de color neutral negro en la parte inferior. Los títeres que al final operan como controladores para la experiencia de interacción son tipo títere de varilla.

\section{Figura 8}

Prototipos y distribución de los componentes de la interfaz del TTH. Imagen cortesía del grupo de investigación Hipertrópico, UdeA.

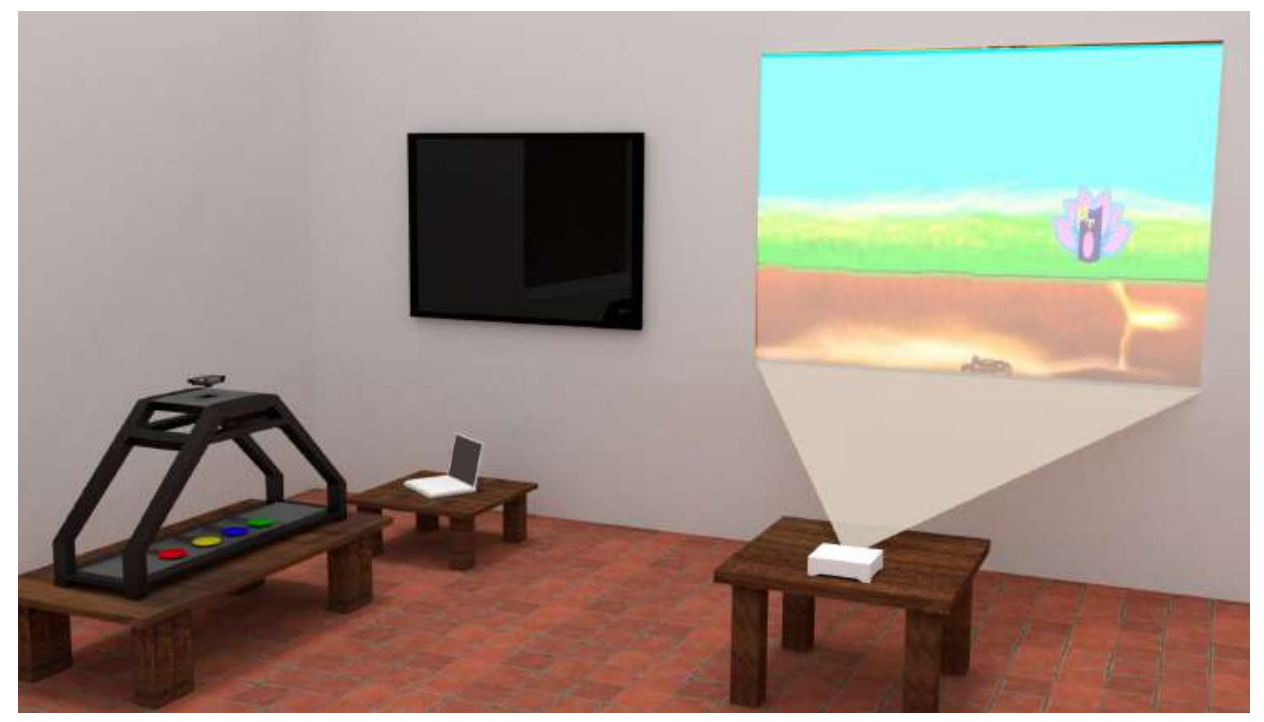

2. Secuencia didáctica de talleres de implementación del teatrino: Para la implementación de la estrategia, se diseñó una secuencia didáctica base que puede ser particularizada acorde con las temáticas, las condiciones de la población y su contexto. Los componentes que estructuran esta secuencia buscan propiciar la construcción de ambientes de aprendizaje y expresión crea- tiva, donde el STEAM emerge tras la articulación de elementos creativos y reflexivos, integrando en este proceso el desarrollo de las diferentes dimensiones del niño. Dentro de estos componentes tenemos: cantidad de sesiones de trabajo, su nombre y duración, objetivos, contenidos orientadores, actividades, habilidades a desarrollar y recursos a utilizar. 


\section{Figura 9}

Creación del escenario / mapa del territorio. Institución Educativa Villa Flora. Imagen cortesía de la investigación Laboratorio de Territorio, Ciudadanía y Paz, desarrollada por el INER y el grupo de investigación Hipertrópico de la Facultad de Artes.

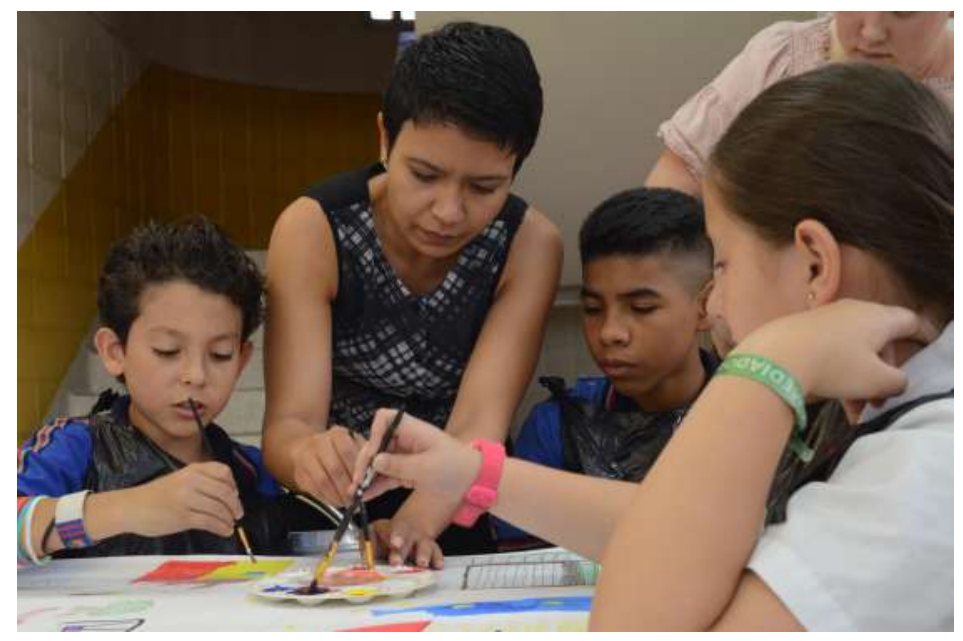

\section{Página web titeresenpantalla.org: el} sitio web se crea como un espacio que favorece el aprendizaje y la expresión creativa. Allí se proponen estrategias de participación a las cuales es posible vincularse creando historias, personajes y escenarios sin que sea condición haber hecho parte de la dinámica de talleres de implementación. Para ello, la persona puede acceder a un formulario en el que se solicitan datos generales de su identificación como autor y de la creación a compartir. Estos datos son recibidos por un equipo de editores que verifica si la información es apta para los niños y niñas a quienes va dirigida la propuesta. Una vez aprobada, se publica el contenido en el sitio web para que otras personas puedan conocer e interactuar con esta creación, fortaleciendo con ello la premisa de intercambio cultural presente en la pregunta de investigación.

\section{Versión expositiva, obra Títeres en} pantalla: El desarrollo de una versión expositiva, tipo instalación, fue pensada en función de poder difundir y llevar la propuesta a otros públicos. Es así como surge la obra
Títeres en pantalla como una instalación dispuesta para que el público interactúe con personajes y escenarios, los cuales han derivado de historias creadas por niños y niñas que fueron digitalizados para configurar la obra.

\section{Figura 10}

Registro: instalación versión expositiva Títeres en Pantalla. Exposición Artware 7, en Lima - Perú, 2018. Imagen cortesía del grupo de investigación Hipertrópico, UdeA.

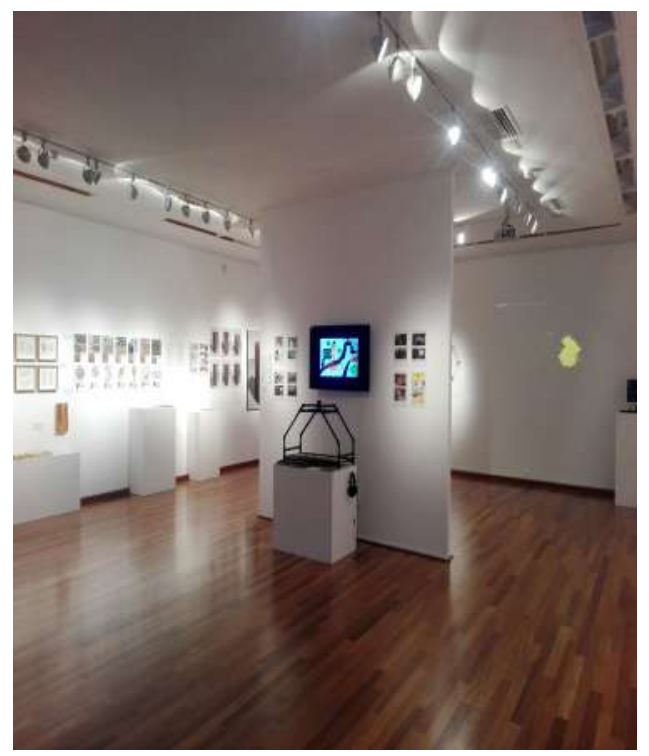




\section{Otros hallazgos}

En el transcurso de la investigación se identificó la participación activa y entusiasta de los niños y niñas como una constante a lo largo de la experiencia. Su actitud atenta y exploración detallada del entorno denotaron interés por el descubrimiento de los espacios físicos a los cuales fueron invitados, así como por el reconocimiento de los materiales para las actividades, la disposición para comprender las situaciones en las cuales se encontraron involucrados y el planteamiento de soluciones acordes a los problemas propuestos. Esta curiosidad evidente a través de la formulación permanente de preguntas mostró que, durante los encuentros, los niños y niñas experimentaron sentimientos de seguridad y tranquilidad, los cuales contribuyeron a la movilización de aprendizajes, a la activación del pensamiento crítico y creativo, $y$ al fortalecimiento de la confianza y el respeto entre participantes, al compartir las opiniones y creaciones resultantes en cada encuentro. De manera particular, estas acciones posibilitaron la expresión creativa, dado que los niños y niñas hicieron uso de su imaginación como recurso para la creación de historias y personajes, acudiendo a sus experiencias y saberes previos para combinar y crear situaciones y elementos totalmente nuevos. También se evidenció que los niños y niñas, en el momento de la creación de historias, se expresaban oralmente de manera fluida al narrar qué significaba cada imagen o qué pasaba en las situaciones imaginadas en sus dibujos; sin embargo, en el momento de la escritura y puesta en escena la expresión oral no ocurría con naturalidad.

Respecto a la convergencia disciplinar STEAM, es posible destacar que los talleres realizados intencionaron el aprendizaje de conceptos clave relacionados con elementos básicos de las matemáticas, tales como dimensiones, conteo, proporción, entre otros. Asimismo, las experiencias propiciaron que los niños y las niñas emprendieran procesos de distinción, clasificación y comparación entre los diferentes objetos, además del acercamiento a las características de los animales y su entorno. Desde la perspectiva tecnológica, el proyecto posibilitó el interés por la interacción con aparatos y la comprensión de algunas de sus funciones con miras a potenciar su utilización como herramienta. Finalmente, a través de diferentes formas de expresión como el dibujo, el sonido, el collage, la producción tridimensional y el movimiento del cuerpo, los niños y niñas se acercaron a las diversas manifestaciones artísticas y su convergencia con medios digitales.

\section{Conclusiones}

En diversas investigaciones referidas a la integración tecnológica en los procesos de enseñanza y aprendizaje se identifica una concentración en el uso de las Tecnologías de la Información y la Comunicación -TIC-
(Jaramillo y Ruíz, 2010; Vega, 2010; Rodríguez, 2004; Reyes y Prado, 2020; González, 2019; Paredes, 2018), el uso de Ambientes Virtuales de Aprendizaje -AVA- (Castiblanco y Díaz, 2010; Páramo, 2010; Florencia y 
Ocampo, 2018; Morado y Ocampo, 2018), y el diseño de software o plataformas para favorecer dichos procesos (Gómez, 2010; Quintero, Munévar y Álvarez, 2009). Son escasas las investigaciones que presentan un acercamiento a la apropiación de recursos tecnológicos (software y hardware) en experiencias de enseñanza y aprendizaje en el campo de las infancias, su relación con las artes y la trascendencia de la interacción o manipulación que los niños y niñas puedan tener con los dispositivos o tecnologías involucradas. Al respecto, a nivel internacional se destaca el aprovechamiento de Makey Makey y Scratch para la expresión musical en la educación inicial (Castro, Ríos y Arguedas, 2020).

A nivel nacional, son pocas las iniciativas que proponen la integración interdisciplinar en experiencias de formación donde los niños y niñas son creadores de historias inspiradas en sus realidades o contextos. Es importante considerar el proyecto Maguaré del Ministerio de Cultura; este es un portal:

"En el que se reúnen más de 600 contenidos entre juegos, canciones, videos, libros y aplicaciones para que los niños de primera infancia, en compañía de un adulto, puedan explorar y divertirse a través de su voz, su cuerpo, su imaginación, sus movimientos y todos sus sentidos". (Maguaré, s.f.)

Moreno (2019) destaca que esta propuesta trata del desarrollo de talleres para que los niños y niñas expresen su cotidianidad a través de historias con las cuales comparten su mundo y sus intereses en la infancia, alejándose de la recepción pasiva para ubicarse en el lugar de los creadores. Este proyecto y las consideraciones de Orozco (2004) presentan a los museos interactivos como escenarios en los que se propician las convergencias disciplinares para el desarrollo de encuentros creativos y pedagógicos que dialogan con los planteamientos de la estrategia Títeres en Pantalla. Todos estos propenden por generar una "experiencia educativa integral y lúdica en la que se desarrollen conocimientos y saberes" a través de "una exploración creativa y una experimentación dirigida que a su vez posibilite un involucramiento intelectual, físico y emocional de sus usuarios" (pp. 1-2). Durante la formulación y desarrollo de la investigación que da lugar a la estrategia Títeres en Pantalla, se visualizan conclusiones a niveles metodológicos, pedagógicos y de diseño, en términos de experiencias educativas integrales que implican la revisión de aspectos relacionados con la implementación, sostenibilidad y transferencia social. Este ejercicio investigativo evidencia la relevancia y pertinencia de los resultados, porque se logra la participación activa de los niños y las niñas en la creación de contenidos escritos e ilustrados, a través de actividades interdisciplinares que, potenciando la expresión creativa, aportan al desarrollo de su creatividad, así como al conocimiento de sus necesidades, intereses y percepciones acerca de su realidad.

En términos metodológicos se encuentra que, a pesar de la poca bibliografía disponible sobre la metodología de investigación-creación, ubicar en ella el desarrollo del trabajo interdisciplinar y de relacionamiento investigativo y creativo, permite desarrollar un proceso que se mueve entre las potencialidades del trabajo académico y el empírico. En particular, con esta metodología se aboga por traducir las reflexiones conceptuales, tecnológicas y metodológicas para la expresión creativa y el aprendizaje holístico localizado, a un pensamiento sensible que se ve formalizado colaborativa y colectivamente en múltiples matices, como: las historias, personajes y escenarios desa- 
rrollados por niños y niñas, los análisis de procesos sistematizados, los planteamientos de dificultades y logros del proceso, el desarrollo de software propio, entre otros.

El diseño de un enunciado de interacción implica diálogos continuos entre especialistas de distintas áreas del conocimiento, y con la comunidad. Es importante tener mayor vínculo en el proceso con profesores de instituciones educativas para identificar el contexto de su práctica docente y las necesidades y perfil de los estudiantes. Desde la perspectiva tecnológica, es importante integrar al equipo un número mayor de ingenieros con el fin de precisar los elementos que facilitan la estabilidad del TTH y sus componentes.

Al finalizar la investigación, aunque la estrategia metodológica queda diseñada $\mathrm{y}$ en potencialidad de uso, contar con una participación real de apropiación fuera de la que se da en la implementación de los talleres implica nuevas etapas de investigación. Esa nueva etapa de investigación, en la que se aborda todo un circuito de socialización pedagógica, el esfuerzo se debe centrar en una especie de posicionamiento mediático que motive el uso y la participación creativa en los distintos espacios de interacción propuestos en el enunciado de interacción de la estrategia Títeres en Pantalla. De esta forma, se haría efectivo el potencial de la pá- gina web como espacio para la creación, el aprendizaje e intercambio creativo, no solo como espacio de difusión.

Las respuestas de los distintos usuarios frente a la estrategia metodológica, sean niños, niñas o adultos, da cuenta del potencial que tiene Títeres en pantalla para facilitar la expresión creativa, el intercambio cultural y el aprendizaje interdisciplinar. En particular, se observa una gran conexión con la plataforma digital, sea en la versión de TTH, en los talleres de implementación del TTH, en www.titeresenpantalla.org o en la obra para ser instalada. Las personas disfrutan el carácter matérico del hacer manual en la creación de las historias, los personajes y escenario, a la vez que se conectan con las representaciones en pantalla que, en algunos casos, son el resultado de sus propias creaciones. Desde el punto de vista performativo, se destaca el nivel de empatía con los personajes y las historias, y la facilidad y compromiso para la relación con los demás, generando interacciones más significativas entre máquinas y humanos.

Agradecimientos: Carlos Mario Sánchez, Diego Mauricio Toro Orlas, Pablo Andrés Pulgarín Herrera, Juan Carlos Hernández Suárez, Katlen Smith Vergara Jiménez, Arney Herrera Herrera, David Alonso Gonzáles Rodríguez y Larry Alberto Vélez.

\section{Notas}

$$
\square
$$

1. Investigación financiada por el Comité para el Desarrollo de la Investigación en la Universidad de Antioquia, CODI, en el año 2015.

2. The research was funded by Comité para el Desarrollo de la Investigación, CODI, in 2015.

3. El concepto de títere es entendido como "El fenómeno que se presenta cuando existe una relación entre un objeto y una fuerza que lo manipula, generalmente humana, y un público que al ser 
testigo de la relación decide compartir la ilusión de una vida imaginada del objeto" (Cárdenas, 2014, p.27.)

4. Implementamos el Taller en este momento de la investigación, ya que implica una organización que integra dinámicas de trabajo individual y grupal en función de una tarea para reflexionar y aprender desde el placer y el juego. Se sustenta "en la participación activa, el constante intercambio, la mirada puesta en lo grupal y la distribución de roles, el trabajo compartido, la producción con otros, la búsqueda de acuerdos y el desarrollo de la autonomía" (Pitluk, 2006, pp 102-103).

5. Según la página principal, "Processing is a flexible software sketchbook and a language for learning how to code within the context of the visual arts. Since 2001, processing has promoted software literacy within the visual arts and visual literacy within technology" (https://processing. org/).

6. El títere de varilla con el cual se controla un personaje a través de un soporte rígido.

7. Se entiende por avatares la representación del títere creado por los niños y niñas en pantalla.

8. Investigación realizada en asociación con el Grupo de Estudios del Territorio, GET, del Instituto de Estudios Regionales, INER.

\section{REFERENCIAS}

Ballesteros, M., y Beltrán, E. (2018). ¿Investigar creando? Una guía para la investigación-creación en la academia. Editorial Universidad del Bosque.

Barreto, M. (2011). Consideraciones ético-metodológicas para la investigación en educación inicial. Revista Latinoamericana de Ciencias Sociales, Niñez y Juventud, 2 (9), 635 - 648

Bourriaud, N (2006). Estética Relacional. Buenos Aires: Adriana Hidalgo editora S.A.

Cárdenas, E. (2014). Materia transformada en títeres. Materia, Forma y Movimiento. La estética del teatro de títeres en Bogotá. Corporación cultural materile. Bogotá: Colombia.

Carmona-Mesa, J. A., Cardona Zapata, M. E., \& Castrillón-Yepes, A. (2020). Estudio de fenómenos físicos en la formación inicial de profesores de Matemáticas. Una experiencia con enfoque STEM. Uni-Pluriversidad, 20 (1), e2020101. https://doi.org/10.17533/udea.unipluri.20.1.02

Castiblanco, A., y Díaz Hortúa, Y. (2010). La red como escenario de socialización: una reflexión sobre los jóvenes y la Mensajería Instantánea. Revista Colombiana de Educación, (58), 140.152. https://doi.org/10.17227/01203916.640

Castro, H., Ríos, K., y Arguedas, C. (2020). Acompañamiento pedagógico del Programa de Tecnologías para el Aprendizaje [Protea]: una experiencia constructivista que aprovecha el Makey Makey y Scratch para enriquecer un curso de expresión musical. Revista Educación, 44 (2). https://www. redalyc.org/jatsRepo/440/44062184001/index.html

Cilleruelo, L., y Zubiaga, A. (2014). Una aproximación a la Educación STEAM. Prácticas educativas en la encrucijada arte, ciencia y tecnología. Revista Jornadas de Psicodidáctica, p. 2. https:// www.augustozubiaga.com/web/wp-content/uploads/2014/11/STEM-TO-STEAM.pdf 
Daza, S. (2009). Investigación - Creación un acercamiento a la investigación en las artes. Horizonte Pedagógico, 11(1), pp. 87-92. Recuperada de: https://revistas.iberoamericana.edu.co/index.php/ rhpedagogicos/article/viewFile/339/303

Florencia, M., y Ocampo, S. (2018). Una experiencia de acompañamiento tecno-pedagógico para la construcción de Entornos Virtuales de Aprendizaje en educación superior. Revista Educación, 43(1) https://www.redalyc.org/jatsRepo/440/44057415004/index.html

Gómez, U. E. (2010). Diseño de un software para favorecer el aprendizaje de estudiantes con necesidades especiales. Revista Colombiana de Educación, (58), 154.170. https://doi. org/10.17227/01203916.641

González, R. (2019). Criterios personalistas para una evaluación antropológica del empleo de las tecnologías en la escuela. Revista Educación, 44(1) https://www.redalyc.org/jatsRepo/440/44060092016/index.html

Guerra, P. (2008) Teatro de Títeres Rito y Metáfora (Trabajo de grado). Universidad de Chile, Santiago de Chile

Herrero, S. (2017). Los títeres en el aula de educación infantil (Trabajo de grado). Universidad de Valladolid, Valladolid.

Jaramillo Marín, P. H., y Ruíz Quiroga, M. (2010). El desarrollo de la autonomía: más allá del uso de las TIC para el trabajo independiente. Revista Colombiana de Educación, (58), 14.39. https:// doi.org/10.17227/01203916.637

Laurel, B. (1993). Computer as Theater. Massachusetts: Addison-Wesley.

Lowenfeld, V. (1961). Desarrollo de la capacidad creadora. Editorial Kapelusz

Maguaré, (s.f.) A cerca de. Dirección de Artes del Ministerio de Cultura. https://maguare.gov.co/ acerca-de/

Morado, M., y Hernández, S. (2018) Una experiencia de acompañamiento tecno-pedagógico para la construcción de Entornos Virtuales de Aprendizaje en educación superior. Revista Educación, 43(1) https://www.redalyc.org/jatsRepo/440/44057415004/index.html

Moreno, E. (2019) Narrativas transmedia, infancia y cultura digital. Infancias Imágenes 18(1) http:// revistas.udistrital.edu.co/ojs/index.php/infancias

Orozco Gómez, G. (2004). Los museos interactivos como mediadores pedagógicos. Revista Colombiana de Educación, (46). https://doi.org/10.17227/01203916.5500

Paredes, W. (2018). Brecha en el uso de tecnologías de la información y comunicación (TIC) básicas y modernas entre estudiantes y docentes en universidades ecuatorianas. Revista Educación, 43(1) https://www.redalyc.org/jatsRepo/440/44057415009/index.html

Pitluk, L. (2006) La planificación didáctica en el Jardín de Infantes. Rosario, Argentina: Homo Sapiens.

Ortiz-Revilla, J., Greca, L., y Arriassecq, I. (2018). Construcción de un marco teórico para el enfoque STEAM en la Educación Primaria en Martínez Losada, C. y García Barros, S. (coord.), 28 Encuentros de Didáctica de las Ciencias Experimentales. Iluminando el cambio educativo. (pp. 823-828). Universidade da Coruña. DOI: https://doi.org/spudc.9788497496896 
Quintero Corzo, J., Munévar Quintero, F. I., y Álvarez Márquez, D. Y. (2009). Ambientes naturales y ambientes virtuales de aprendizaje. Revista Colombiana de Educación, (56). https://doi. org/10.17227/01203916.7579

Reina, C. (2009). El teatro infantil. Revista digital de Innovación y experiencias educativas, $\mathrm{n}^{\circ} 15$ (45), pp (1-13) Recuperada de: https:/archivos.csif.es/archivos/andalucia/ensenanza/revistas/ csicsif/revista/pdf/Numero_15/CRISTINA_REINA_2.pdf

Restrepo, I. (2014). Tocar con los ojos, ver con las manos. Cuerpo, acción y tecnología en los espacios de interacción digital con monitoreo remoto (Tesis doctoral). Doctorado en Artes, Universidad de Antioquia.

Reyes, R., y Prado, A. (2020) Las Tecnologías de Información y Comunicación como herramienta para una educación primaria inclusiva. Revista Educación, 44(2). https://www.redalyc.org/jatsRepo/440/44062184011/index.html

Rodríguez, J. (2004). Medios y tecnologías de la información y la comunicación: una caracterización de las prácticas en instituciones escolares de Bogotá. Revista Colombiana de Educación, (46). https://doi.org/10.17227/01203916.5506

Stern, A. (1977). La expresión. Barcelona, España: Promoción cultural.

Stern, A. (2008). Del dibujo infantil a la semiología de la expresión. Iniciación a otra mirada sobre el trazo. $\left(3^{a} e d\right)$. Cataluña, España: Carena

Vega, J. R. (2010). Las TIC y el aprendizaje de las lenguas extranjeras. Revista Colombiana de Educación, (58), 78.95. https://doi.org/10.17227/01203916.636

Vigotsky, L. (2009) Imaginación y creatividad en la infancia. 9a edición. Madrid: Akal. 